\title{
Nuclear Physics on the Lattice
}

\section{Tetsuo HATSUDA*}

Physics Department, The Univ. of Tokyo, Tokyo 113-0033, Japan

IPMU, The Univ. of Tokyo, Kashiwa 277-8583, Japan

E-mail: hatsuda@phys.s.u-tokyo.ac.jp

After a brief introduction to the phenomenological nuclear force and its relevance to nuclear physics, we review the recent development of the nucleon-nucleon force derived from the quenched and (2+1)-flavor lattice QCD simulations. Interactions among octet-baryons in the flavor SU(3) limit are also discussed on the basis of the 3-flavor lattice QCD simulations to unravel the origin of the nuclear repulsive core and the possible existence of the $H$-dibaryon.

The XXVIII International Symposium on Lattice Field Theory, Lattice2010

June 14-19, 2010

Villasimius, Italy

${ }^{*}$ Speaker. 


\section{Introduction}

Understanding of the nuclear force from quantum chromodynamics (QCD) is one of the most challenging problems in nuclear and particle physics. Experimentally, a large number of protonproton and neutron-proton scattering data as well as deuteron properties have been accumulated and summarized e.g. in the Nijmegen database [1]. Below the pion production threshold, the notion of the $N N$ potential (either in the coordinate space or in the momentum space) is useful in the sense that it can be used not only to describe the two-body system but also to study the nuclear many-body problems through ab-initio calculations [2].

The phenomenological $N N$ potentials in the coordinate space are known to reflect some characteristic features of the $N N$ interaction [3]:

(i) The long range part of the nuclear force (the relative distance $r>2 \mathrm{fm}$ ) is dominated by the one-pion exchange [4]. Because of the pion's Nambu-Goldstone character, it couples to the spinisospin density of the nucleon and hence leads to the tensor force.

(ii) The medium range part ( $1 \mathrm{fm}<r<2 \mathrm{fm}$ ) receives significant contributions from the exchange of multi-pions. In particular, the spin-isospin independent attraction of about $50-100 \mathrm{MeV}$ in this region plays an essential role to nuclear binding.

(iii) The short range part $(r<1 \mathrm{fm})$ is best described by a strong repulsive core [5]. Such a short range repulsion is relevant for the maximum mass of neutron stars.

(iv) There is also a strong attractive spin-orbit force in the isospin 1 channel at medium and short distances. This leads to the ${ }^{3} \mathrm{P}_{2}$ neutron pairing in neutron matter and hence the neutron superfluidity inside neutron stars [7].

Several high precision $N N$ forces are now available to fit neutron-proton and proton-proton scattering data (about 4500 data points) with $\chi^{2} /$ dof $\sim 1$. However, they have typically 20-40 fitting parameters: e.g. CD Bonn potential, AV18 potential and $\mathrm{N}^{3} \mathrm{LO}$ chiral effective field theory have 38, 40, and 24 parameters, respectively [8]. If one tries to extend these to hyperon-nucleon and hyperon-hyperon interactions, the task becomes extremely tough since the number of parameters increase and the scattering data are scarce. In this situation, it is highly desirable to study the general baryon-baryon interactions from the first principle lattice QCD simulations, since all the hadronic interactions in QCD are controlled only by the QCD scale parameter $\left(\Lambda_{\mathrm{QCD}}\right)$ and the quark masses $\left(m_{u}, m_{d}, m_{s}\right)$ whose values are pretty well determined [9].

A theoretical framework to study the hadron-hadron interaction using lattice QCD was first proposed by Lüscher [10] and was applied to the lattice QCD simulations for the $N N$ interaction in [11]: For two hadrons in a finite box with a size $L \times L \times L$ in the periodic boundary condition, an exact relation between the energy spectra in the box and the elastic scattering phase shift at these energies was derived. If the range of the hadronic interaction $R$ is sufficiently smaller than the size of the box $R<L / 2$, the behavior of the equal-time Nambu-Bethe-Salpeter (NBS) amplitude $\psi(r)$ in the interval $R<|r|<L / 2$ under the periodic boundary condition has sufficient information to relate the phase shift and the two-particle spectrum. This Lüscher's method bypasses the difficulty to treat the real-time scattering process on the Euclidean lattice.

Recently, an alternative approach to the hadron interactions in lattice QCD was proposed [12, 13, 14. The starting point is the same equal-time NBS amplitude $\psi(r)$ : Instead of looking at the amplitude outside the range of the interaction, the internal region $|r|<R$ is considered and an 
energy-independent non-local potential $U\left(r, r^{\prime}\right)$ is defined from $\psi(r)$. Since $U\left(r, r^{\prime}\right)$ in QCD is a localized function in space due to confinement of quarks and gluons, it receives finite volume effect only weakly. Therefore, once $U$, although it is not a direct physical observable, is determined on the lattice, one may simply use the Schrödinger equation in the infinite space to calculate observables such as the scattering phase shifts, bound state spectra etc. Moreover, the potential would be a smooth function of the quark masses: This is in sharp contrast to the scattering length which shows a singular behavior around the quark mass corresponding to the formation of the two-body bound states such as the deuteron. Similar situation is well-known in the BEC-BCS crossover of cold fermionic atoms [15].

In this article, we will eview some recent results of the nuclear force on the lattice (or the lattice nuclear force in short) after a brief introduction to the basic formulation.

\section{Deriving the $N N$ potential on the lattice}

\subsection{NBS wave function on the lattice}

In field theory, the best analogue of the two-particle wave function is the equal-time NambuBethe-Salpeter (NBS) amplitude or the"NBS wave function": Let us consider an exact six-quark state $|\mathscr{E}\rangle$ which has total energy $\mathscr{E}$, total three-momentum zero and total electric charge $+e$ in a finite box. Then we define the NBS wave function by

$$
\psi(r)=\left\langle 0\left|n_{\beta}(x+r, t=0) p_{\alpha}(x, t=0)\right| \mathscr{E}\right\rangle .
$$

The local composite operators for the proton and the neutron are denoted by $p_{\alpha}(x, t)$ and $n_{\beta}(y, t)$ with spinor indices $\alpha$ and $\beta$. One should keep in mind that $|\mathscr{E}\rangle$ is not a simple superposition of a product state $|\mathrm{p}\rangle \otimes|\mathrm{n}\rangle$, since there are complicated exchanges of quarks and gluons between the two composite particles. The NBS wave function $\psi(r)$ can be regarded as a probability amplitude in $|\mathscr{E}\rangle$ to find "neutron-like" three-quarks located at point $x+r$ and "proton-like" three-quarks located at point $x$.

The spatial extent of the $N N$ interaction in QCD is short ranged and is exponentially suppressed beyond the distance $R \sim 2 \mathrm{fm}$. Therefore, the spatial part of the NBS wave function in the "outer region" satisfies the Helmholtz equation,

$$
\left(\nabla^{2}+k^{2}\right) \psi(r)=0 \quad(|r|>R),
$$

up to an exponentially small correction in $L$. Here the "asymptotic momentum" $k$ is determined by the asymptotic behavior of the wave function in the outer region.

An important property of the NBS wave function $\psi(r)$ is that its asymptotic behavior at large $|r|$ in the infinite volume limit reproduces the correct phase shift obtained from the $S$-matrix of the elastic $N N$ scattering. This can be shown explicitly by using the Nishijima-ZimmermannHaag(NHZ)'s reduction formula [16] for the products of local composite operators. (The proof is given in Appendix A of [14].) To define the NBS wave function on the lattice, we start with the four-point function

$$
\mathscr{G}\left(r, t-t_{0}\right)=\left\langle 0\left|n_{\beta}(x+r, t) p_{\alpha}(x, t) S\left(t_{0}\right)\right| 0\right\rangle \rightarrow \psi(r) e^{-\mathscr{E}_{0}\left(t-t_{0}\right)}\left(t \gg t_{0}\right),
$$

where $\mathscr{E}_{0}$ is the lowest energy state created by the source operator $S\left(t_{0}\right)$. 


\subsection{Non-local potential and the velocity expansion}

To define the $N N$ potential from the NBS wave function, let us introduce the following local function:

$$
K_{E}(r)=\frac{1}{2 \mu}\left(\nabla^{2}+k^{2}\right) \psi_{E}(r) \equiv\left(E-H_{0}\right) \psi_{E}(r)
$$

In the second equality, we introduce an "effective center of mass energy", $E=k^{2} /(2 \mu)$, and the free Hamiltonian $H_{0}=-\nabla^{2} /(2 \mu)$, with $\mu=m_{N} / 2$ being the reduced mass of the two nucleons. They are introduced only to make a formal resemblance with the Schrödinger type equation and have nothing to do with non-relativistic approximation. Hereafter, we put the suffix $E$ to the NBS wave function to emphasize its $E$-dependence. Since the "plane-wave" part of the NBS wave function in the outer region $(r>R)$ is projected away by the operator $E-H_{0}$, the function $K_{E}(r)$ is non-vanishing only in the inner region $(r<R)$. Note also that the Fourier transform of $K_{E}(r)$ is essentially the half off-shell $T$-matrix.

We can rewrite Eq. (2.4) in two equivalent ways:

$$
\left(E-H_{0}\right) \psi_{E}(r)=U_{E}(r) \psi_{E}(r)=\int U\left(r, r^{\prime}\right) \psi_{E}\left(r^{\prime}\right) d r^{\prime} .
$$

The first equality is just a definition of the energy-dependent local potential, $U_{E}(r)=K_{E}(r) / \psi_{E}(r)$. On the other hand, the energy-independent non-local potential, $U\left(r, r^{\prime}\right)$, is defined from $U_{E}(r)$ through a self-consistent equation,

$$
U\left(r, r^{\prime}\right)=\left\langle r|\hat{U}| r^{\prime}\right\rangle=\sum_{E} \int_{-\infty}^{+\infty} \frac{d t}{2 \pi} U_{E}(r)\left\langle r\left|e^{i\left(\hat{H}_{0}+\hat{U}-E\right) t}\right| r^{\prime}\right\rangle .
$$

Carrying out the $t$ integration formally, one may also write Eq. (2.6) as

$$
\hat{U}=\sum_{E} \hat{U}_{E} \boldsymbol{\delta}\left(E-\hat{H}_{0}-\hat{U}\right)
$$

In these formulas, $\sum_{E}$ stands for the summation (integration) over the discrete (continuum) energies. In particular, $E$ is always discrete on the lattice with a finite volume. Also, $E$ has an upper limit $E_{c}$ at which inelastic scattering starts to take place. Eliminating the $E$-dependence of the potential through Eq. (2.6) has been discussed in a transparent manner by Królikowski and Rzewuski [17] long time ago: their motivation was to prove the equivalence between the multiple-time NambuBethe-Salpeter type equation with an $E$-dependent kernel and the equal-time Schrödinger type equation with an $E$-independent potential. Essentially the same method was rediscovered and discussed in [12, 13, 14] in the context of the NBS wave function on the lattice.

If we further focus on the low-energy scattering with $E$ sufficiently smaller than the intrinsic scale of the system or the scale of the non-locality of the potential in Eq. 2.5, the velocity expansion of $U\left(r, r^{\prime}\right)$ in terms of its non-locality is useful [18]: For example, the potential with hermiticity, rotational invariance, parity symmetry, and time-reversal invariance may be expanded as [19]

$$
\begin{aligned}
& U\left(r, r^{\prime}\right)=V(r, v) \boldsymbol{\delta}\left(r-r^{\prime}\right), \\
& V(r, v)=\underbrace{V_{C}(r)+V_{T}(r) S_{12}}_{\mathrm{LO}}+\underbrace{V_{L S}(r) L \cdot S}_{\mathrm{NLO}}+\underbrace{O\left(v^{2}\right)}_{\mathrm{N}^{2} \mathrm{LO}}+\cdots,
\end{aligned}
$$


where $v=p / \mu$ and $L=r \times p$ with $p=-i \nabla$, and

$$
S_{12}=\frac{3}{r^{2}}\left(\sigma_{1} \cdot r\right)\left(\sigma_{2} \cdot r\right)-\sigma_{1} \cdot \sigma_{2}
$$

Each coefficient of the expansion is a local potential and can be determined successively by measuring the NBS wave functions for several different energies. The central potential $V_{C}$ and the tensor potential $V_{T}$ are classified as the leading order (LO) potentials since they are of $O\left(v^{0}\right)$. The nextto-leading (NLO) potential of $O(v)$ is the spin-orbit potential $V_{L S}(r)$. The LO and NLO potentials are phenomenologically known to be the dominant interactions at low energies.

An advantage of defining the potential from the NBS wave functions in the "inner region" is that the effect of the lattice boundary is exponentially suppressed for finite range interactions: Then one can first make appropriate extrapolation of $U\left(r, r^{\prime}\right)$ or $V(r, v)$ to $L \rightarrow \infty$, and then solve the Schrödinger equation using the extrapolated potential to calculate the observables such as the phase shifts and binding energies in the infinite volume. This is in contrast to the Lüscher's approach [10] in which the wave functions in the "outer region" suffering from the boundary conditions is ingeniously utilized to probe the scattering observables. Apparently, the two approaches are the opposite sides of a same coin.

\subsection{Interpolating operator and the potential}

In Eq. 2.1], simplest interpolating operators for the neutron and the proton written in terms of the up-quark $u(x)$ and the down-quark $d(x)$ would be

$$
n_{\beta}(x)=\varepsilon_{a b c}\left(u_{a}(x) C \gamma_{5} d_{b}(x)\right) d_{c \beta}(x), p_{\alpha}(x)=\varepsilon_{a b c}\left(u_{a}(x) C \gamma_{5} d_{b}(x)\right) u_{c \alpha}(x),
$$

where $x=(x, t)$ and the color indices are denoted by $a, b$ and $c$. The charge conjugation matrix in the spinor space is denoted by $C$. The local operators given above are most convenient for relating the NBS wave function to the four-point Green's function and the scattering observables at $L \rightarrow \infty$ through the NZH reduction formula.

In principle, one may choose any composite operators with the same quantum numbers as the nucleon to define the NBS wave function. Different interpolating operators lead to different NBS wave functions and different $N N$ potentials. However, they lead to the same physical observables by construction. Analogous situation can be seen in quantum mechanics where the unitary transformations modify both the wave function and the potential in such a way that observables are unchanged. Even more direct analogy is in field theory for point-like particles: Field re-definitions modify the vertices and propagators in the Feynmann rule, while the on-shell $S$-matrix is not affected by such changes.

\subsection{Central and tensor forces}

In the $\mathrm{LO}$ of the velocity expansion in Eq. 2.9, we have the central potential $V_{C}(r)$ and the tensor potential $V_{T}(r)$, so that the Shrödinger equation reads

$$
\left(E-H_{0}\right) \psi_{E}(r)=\left(V_{C}(r)+V_{T}(r) S_{12}\right) \psi_{E}(r) .
$$

The central potential acts separately on the orbital S-state and the D-state, while the tensor potential provides a coupling between these two. Therefore, a coupled-channel Schrödinger equation is 

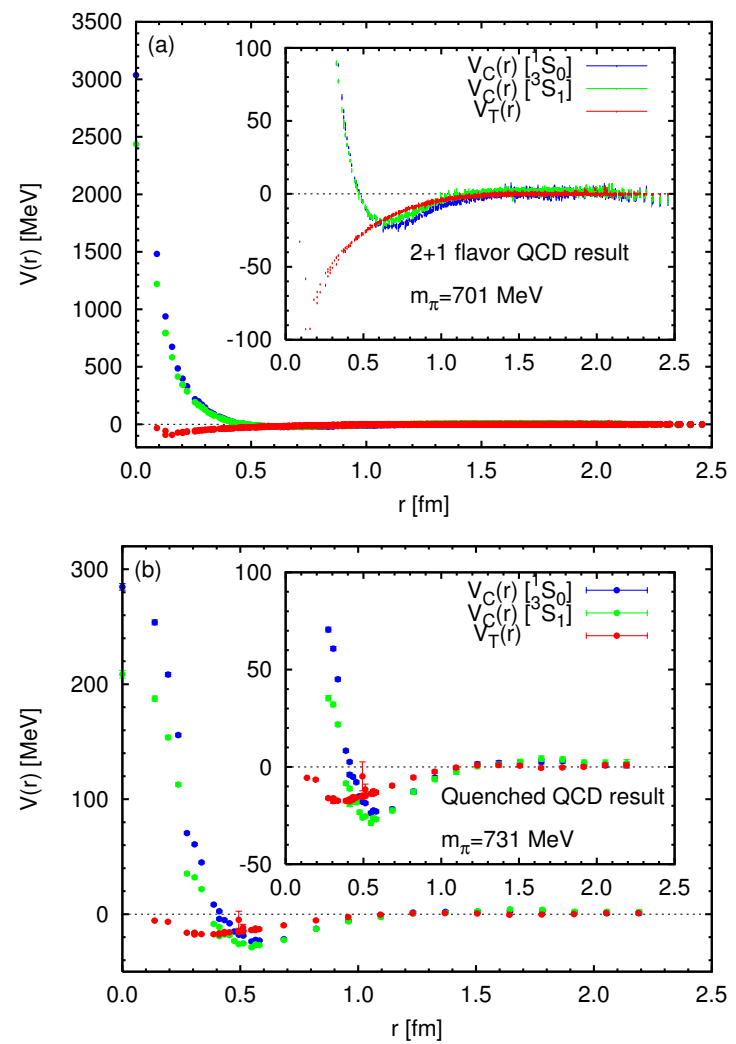

Figure 1: (a) LO potentials in (2+1)-flavor QCD for $m_{\pi}=701 \mathrm{MeV}$ [22]. (b) LO potentials in quenched QCD for $m_{\pi}=731 \mathrm{MeV}$ [14].

obtained from Eq.(2.12) by using the projection operators $\mathscr{P}$ and $\mathscr{Q}$ to the S-state and D-state, respectively. Eventually we calculate $V_{C}$ and $V_{T}$ from the following formula where the quantities in the right hand side are all known on the lattice:

$$
\left(\begin{array}{c}
V_{C} \\
V_{T}
\end{array}\right)=\left(\begin{array}{cc}
\mathscr{P} \psi_{E} & \mathscr{P} S_{12} \psi_{E} \\
\mathscr{Q} \psi_{E} & \mathscr{Q} S_{12} \psi_{E}
\end{array}\right)^{-1}\left(\begin{array}{cc}
E-H_{0} & 0 \\
0 & E-H_{0}
\end{array}\right)\left(\begin{array}{c}
\mathscr{P} \psi_{E} \\
\mathscr{Q} \psi_{E}
\end{array}\right) .
$$

\section{Numerical results in quenched and full QCD simulations}

\subsection{LO potentials}

To demonstrate whether the formalism discussed in the previous section indeed works, we first carried out a quenched QCD simulations with the standard plaquette gauge action and the standard Wilson quark action on a $32^{3} \times 48$ lattice [12, 13, 14]. The lattice spacing is $a=0.137$ $\mathrm{fm}$ which corresponds to the spatial size $L=4.4 \mathrm{fm}$. The light quark masses are chosen so that we have $m_{\pi}=731,529$ and $380 \mathrm{MeV}$ and $m_{N}=1558,1334$ and $1197 \mathrm{MeV}$, respectively. Periodic or anti-periodic boundary conditions are imposed on the quark field along the spatial direction.

As for full QCD, we use the PACS-CS gauge configurations in (2+1)-flavor QCD generated by the Iwasaki gauge action and the $O(a)$-improved Wilson quark (clover) action on a $32^{3} \times 64$ 

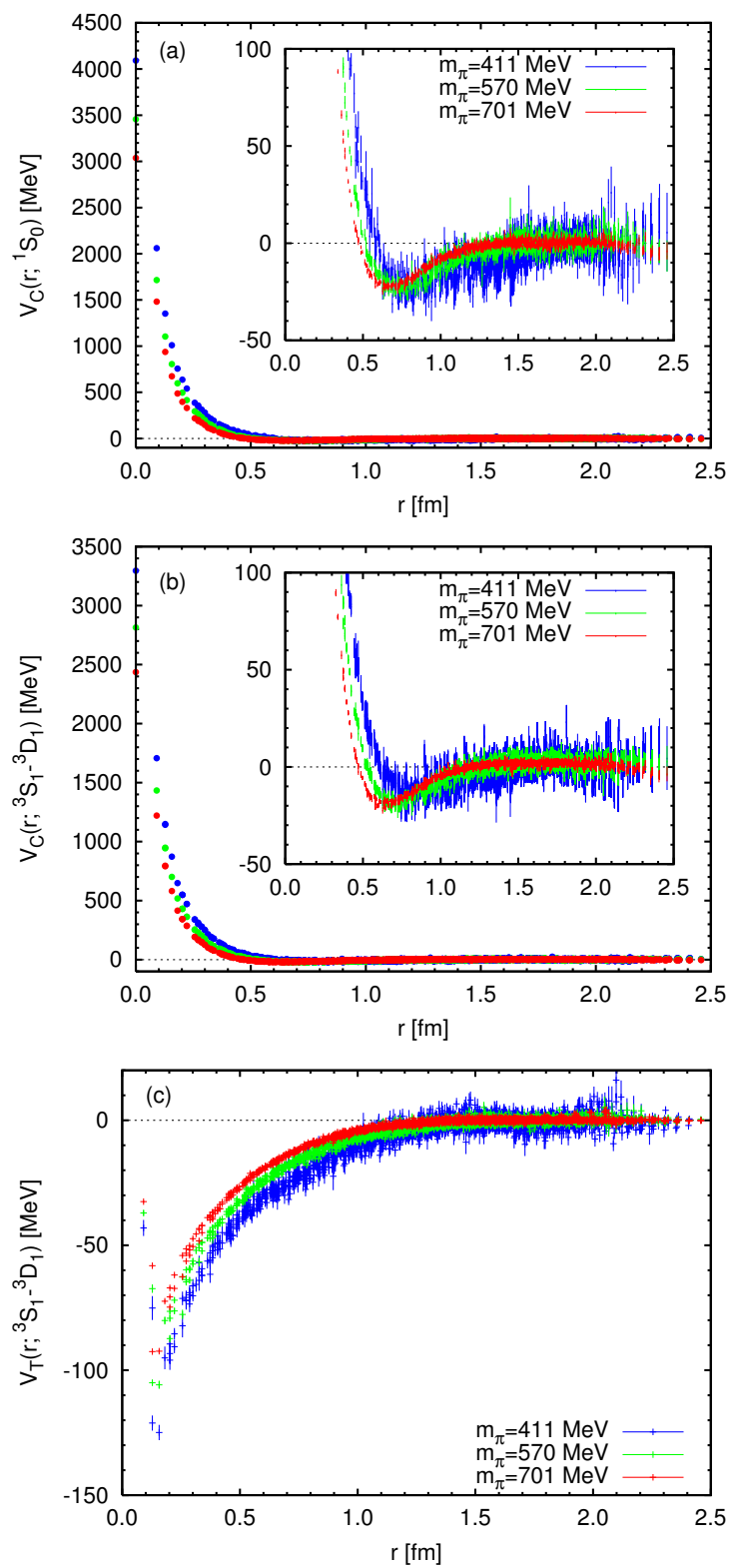

Figure 2: Quark mass dependence of the LO potentials in (2+1)-flavor QCD. (a) The central potential in the spin-singlet channel, (b) the central potential in the spin-triplet channel, and (c) the tensor potential in the spin-triplet channel [22].

lattice [21]. The lattice spacing is $a=0.091 \mathrm{fm}$ which corresponds to the spatial size $L=2.9 \mathrm{fm}$. The light quark masses are chosen so that we have $m_{\pi}=701,570$ and $411 \mathrm{MeV}$ and $m_{N}=1583$, 1412 and $1215 \mathrm{MeV}$, respectively. Also, Periodic boundary condition is imposed on the quark field along the spatial direction.

Shown in Fig 1 a) are the LO potentials $\left(V_{C}\right.$ for ${ }^{1} S_{0}$ and ${ }^{3} S_{1}$ channels and $V_{T}$ determined from ${ }^{3} S_{1}-{ }^{3} D_{1}$ channel) in (2+1)-flavor QCD for $m_{\pi}=701 \mathrm{MeV}$. Even with such a large quark mass, there is a clear evidence of the repulsive core surrounded by attractive well for for central potential and 

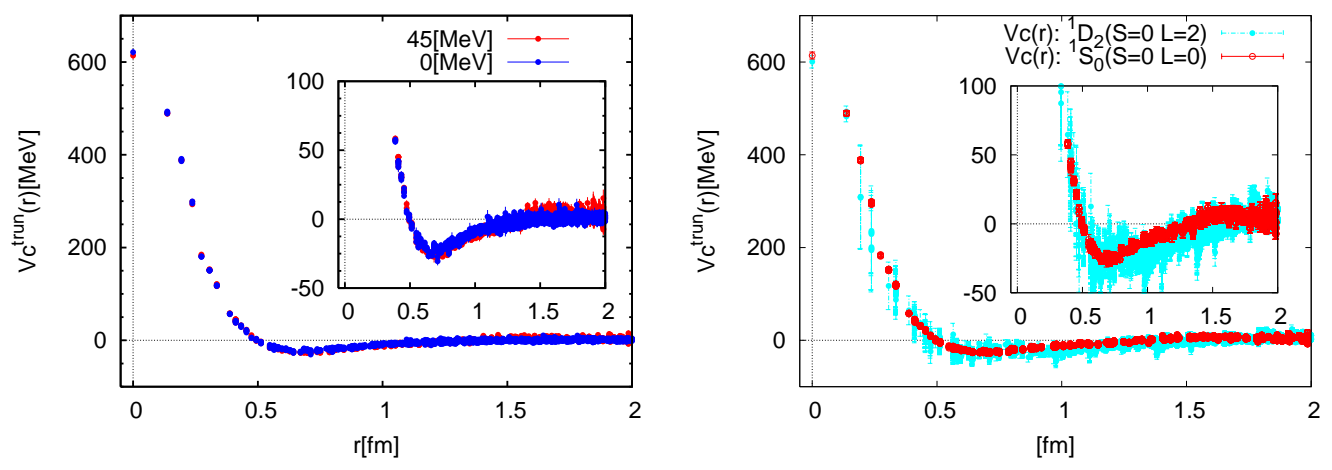

Figure 3: (Left) A comparison of the ${ }^{1} S_{0}$ central potentials obtained at different energies ( $E \sim 0 \mathrm{MeV}$ vs. $E \sim 45 \mathrm{MeV}$ ). (Right) A comparison of the spin-singlet central potential at $E \sim 0 \mathrm{MeV}$ with different orbital angular momenta ( $L=0$ vs. $L=2$ ). Figures are taken from [23].

an evidence of a mild tensor force [22]. They have qualitative similarity with phenomenological potentials. We show in Fig, (1) the LO potentials in quenched QCD with $m_{\pi}=731 \mathrm{MeV}$ for comparison [14]. Although the qualitative structure of the potentials are the same, the magnitude of the repulsive core and the tensor force are relatively weak in quenched QCD.

Shown in Fig $2(a, b, c)$ are the quark mass dependence of the LO potentials in $(2+1)$-flavor QCD [22]. As the quark mass decreases, the repulsive core in $(a, b)$ and the tensor force in (c) become stronger and the attractive well in $(a, b)$ becomes wider. We have fitted these potentials and have calculated the $N N$ scattering phase shift by solving the Schrödinger equation. We found that deuteron bound state does not appear for these quark masses, so that further reduction of the quark mass would be necessary to obtain the realistic lattice potentials.

\subsection{Convergence of the velocity expansion}

So far, the potentials are derived with the periodic boundary condition in the spatial direction for the quark fields. This leads to the the "effective center of mass energy" $E=k^{2} /(2 \mu)$ almost zero. To study the convergence of the velocity expansion of the non-local potential in Eq.2.9, we compare the local ${ }^{1} S_{0}$ potential (in quenched QCD with $m_{\pi}=529 \mathrm{MeV}$ ) obtained at $E \simeq 0 \mathrm{MeV}$ under the periodic boundary condition and that obtained at $E \simeq 45 \mathrm{MeV}$ under the anti-periodic boundary condition [23]. Good agreement between the two as shown in the left panel of Fig 3 indicates that a $O\left(v^{2}\right)$ term in the $\mathrm{N}^{2} \mathrm{LO}$ level is rather small in this energy interval. Shown in the right panel of Fig 3 is a test for a different $O\left(v^{2}\right)$ term in the $\mathrm{N}^{2} \mathrm{LO}$ level [23]: In this case, local potentials determined at the same energy $(E \simeq 0 \mathrm{MeV})$ with different orbital angular momenta $(L=0,2)$ in the spin-singlet channel are compared. Again, within statistical errors, the effect of the $\mathrm{N}^{2} \mathrm{LO}$ term is likely to be small.

\section{Hyperon interactions}

To unravel the origin of the repulsive core in the $N N$ interaction, let us consider the $S$-wave interaction between octet baryons in the flavor SU(3) limit. In this case, two baryon states with a 

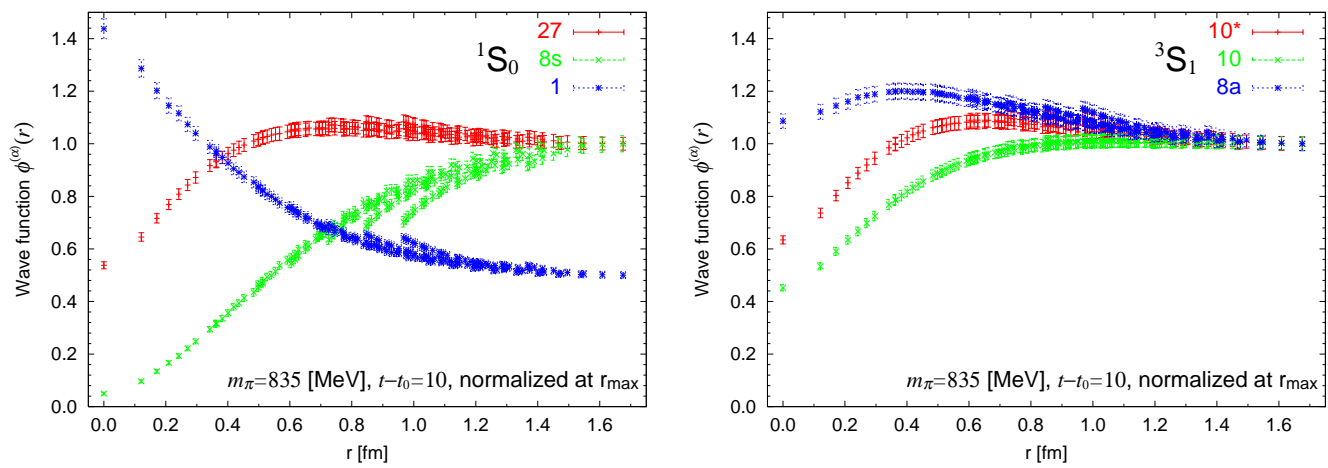

Figure 4: NBS wave functions at $m_{\pi}=835 \mathrm{MeV}$, normalized to $1 / 2$ for the singlet channel and to 1 for other channels at the maximum distance [25.

given angular momentum are labeled by the irreducible flavor multiplets as

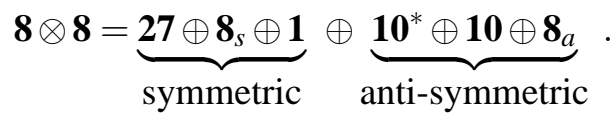

Here "symmetric" and "anti-symmetric" stand for the symmetry under the flavor exchange of two baryons. For the system in the orbital S-wave, the Pauli principle between two baryons imposes $\mathbf{2 7}, \boldsymbol{8}_{s}$ and $\mathbf{1}$ to be spin singlet $\left({ }^{1} S_{0}\right)$ while $10^{*}, \mathbf{1 0}$ and $\mathbf{8}_{a}$ to be spin triplet $\left({ }^{3} S_{1}\right)$. Since there are no mixings among different multiplets in the SU(3) limit, one can define the corresponding potentials as

$$
\begin{aligned}
& { }^{1} S_{0}: V^{(\mathbf{2 7})}(r), V^{\left(\mathbf{8}_{s}\right)}(r), V^{(\mathbf{1})}(r), \\
& { }^{3} S_{1}: V^{\left(\mathbf{1 0}^{*}\right)}(r), V^{(\mathbf{1 0})}(r), V^{\left(\mathbf{8}_{a}\right)}(r) .
\end{aligned}
$$

Potentials among octet baryons, both the diagonal part $\left(B_{1} B_{2} \rightarrow B_{1} B_{2}\right)$ and the off-diagonal part $\left(B_{1} B_{2} \rightarrow B_{3} B_{4}\right)$, are obtained by suitable combinations of $V^{(\alpha)}(r)$ with $\alpha=\mathbf{2 7}, \mathbf{8}_{s}, \mathbf{1}, \mathbf{1 0}, \mathbf{1 0}, \mathbf{8}_{a}$.

In this SU(3) study, we employ the gauge configurations on a $16^{3} \times 32$ lattice generated by CPPACS and JLQCD Collaborations with the renormalization group improved Iwasaki gauge action and the non-perturbatively $O(a)$ improved Wilson quark action. The lattice spacing and the lattice volume are $a=0.121(2) \mathrm{fm}$ and $L=1.93(3) \mathrm{fm}$, respectively. These configurations are provided by Japan Lattice Data Grid (JLDG) and International Lattice Data Grid (ILDG) [24].

Fig. 4 shows the NBS wave functions as a function of the relative distance between two baryons at $m_{\pi}=835 \mathrm{MeV}$ [25]. To draw all data in a same scale, they are normalized to $1 / 2$ for the singlet channel and to 1 for other channels at the maximum distance. The wave functions in Fig. 4 show characteristic flavor dependence: In particular, a strong suppression at short distance appears in the $\mathbf{8}_{s}$ channel, while a strong enhancement appears in the $\mathbf{1}$ channel. Similar results are obtained for $m_{\pi}=1014 \mathrm{MeV}$ too.

Fig. 5] shows the resulting three independent BB potentials in the ${ }^{1} S_{0}$ channel in the flavor basis obtained from the NBS wave functions. Red bars (green crosses) data correspond to the pion mass $1014 \mathrm{MeV}$ ( $835 \mathrm{MeV}$ ): Although there is a tendency that the magnitude (range) of the 

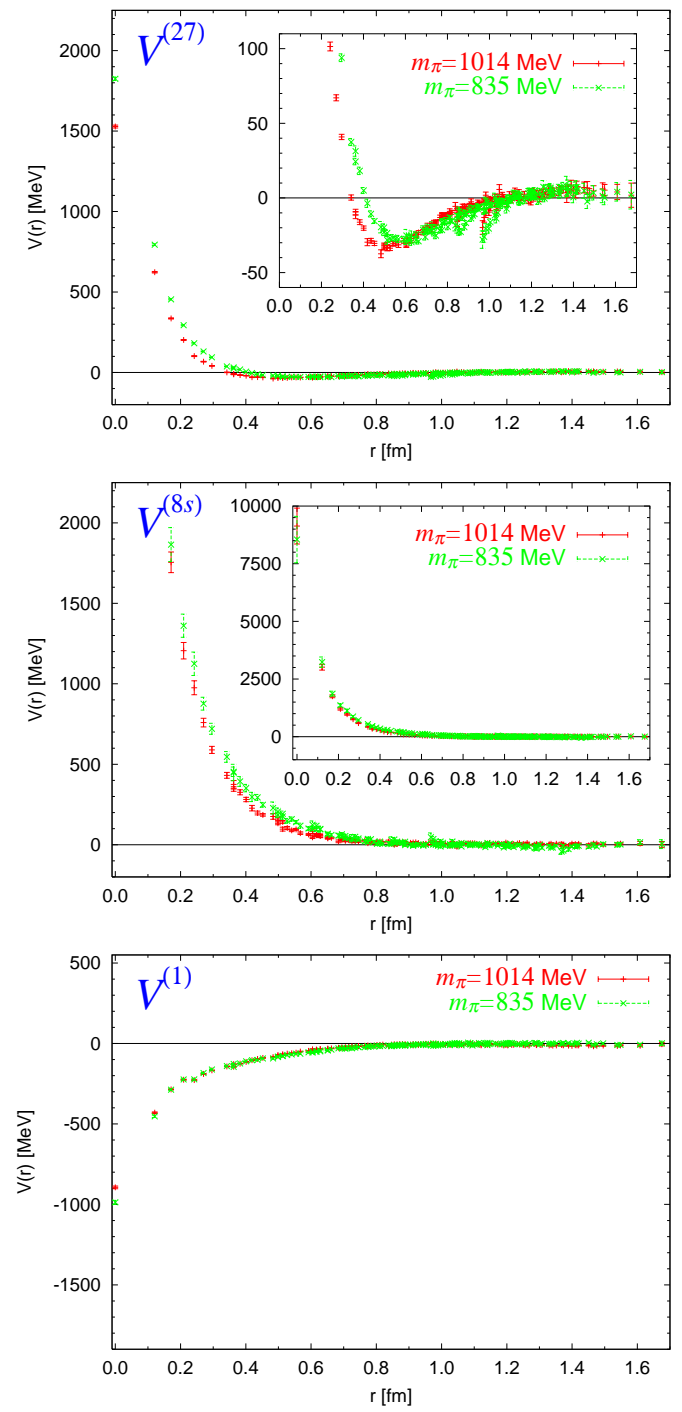

Figure 5: The three independent BB potentials in the ${ }^{1} S_{0}$ channel in the flavor SU(3) limit, extracted from the lattice QCD simulation at $m_{\pi}=1014 \mathrm{MeV}$ (red bars) and $m_{\pi}=835 \mathrm{MeV}$ (green crosses).

potentials becomes larger at short distances (longer at large distances) for lighter quark mass, the differences are not substantial for the present heavy quark masses. Left panels of Fig. [5] show $V^{(27)}$ which corresponds to $\mathrm{NN}{ }^{1} S_{0}$ potential. It has a repulsive core at short distance and an attractive pocket as we have shown already in quenched and $(2+1)$-flavor simulations. The middle panel of Fig. 5 corresponding to $V^{\left(\boldsymbol{8}_{s}\right)}$ has a very strong repulsive core among all channels. In contrast, the right panel of Fig. 5 corresponding to $V^{(\mathbf{1})}$ shows attraction for all distances, which is relevant to the cereblated $H$-dibaryon [26].

These features are consistent with what has been observed in phenomenological quark models [27]. In particular, the potential in the $\mathbf{8}_{s}$ channel in quark models becomes strongly repulsive at short distance since the six quarks cannot occupy the same orbital state due to quark Pauli blocking. On the other hand, the potential in the $\mathbf{1}$ channel does not suffer from the quark Pauli 
blocking and can become attractive due to short range gluon exchange. Such an agreement between the lattice data and the phenomenological models indicates that the quark Pauli blocking plays an essential role for the repulsive core in BB systems as suggested long time ago in [28]. One can also confirm the idea of the Pauli blocking by considering the meson-baryon interaction such as charmonium-nucleon potential [29] and kaon-nucleon potential [30] within the present lattice approach. Generalization of the baryon-baryon interaction to the case with explicit SU(3) breaking is also under way [31].

\section{Summary and concluding remarks}

In this article, we have discussed the basic notion of the nucleon-nucleon potential and its field-theoretical derivation from the equal-time Nambu-Bethe-Salpeter wave function in QCD. By construction, the non-local potential defined through the projection of the wave function to the interaction region (the inner region) correctly reproduces the asymptotic form of the wave function in the region beyond the range of the nuclear force (the outer region). Thus the observables such as the phase shifts and the binding energies can be calculated after extrapolating the potential to the infinite volume limit. Non-locality of the potential can be taken into account successively by making its velocity expansion, which introduces the velocity-dependent local potentials. The leading-order terms of such velocity expansion for the nucleon-nucleon interaction are the central and the tensor potentials.

Some results in the quenched and (2+1)-flavor lattice QCD simulations are shown for relatively heavy pion masses, $m_{\pi} \sim 400,500,700 \mathrm{MeV}$. We found that the $N N$ potential calculated on the lattice at low energy shows all the characteristic features expected from the empirical $N N$ potentials obtained from the experimental $N N$ phase shifts, namely the attractive well at long and medium distances and the repulsive core at short distance for the central potential. As for the tensor potential obtained from the coupled channel treatment of the ${ }^{3} S_{1}$-state and the ${ }^{3} D_{1}$-state, we found appreciable attraction at long and medium distances.

As the quark mass decreases, the repulsive core and attractive well in the central potential, and the attractive well in the tensor potential tend to be enhanced. To make the deuteron bound state, however, it is necessary to go the lighter quark masses. We have also shown that the derivative expansion in terms of the local and energy-independent potentials works well at low energies for at least the quark masses studies above.

There are a number of directions to be investigated on the basis of our approach. Among others, the most important direction is to carry out $(2+1)$-flavor simulations with a large volume (e.g. $L=6 \mathrm{fm})$ at physical quark mass $\left(m_{\pi}=135 \mathrm{MeV}\right)$ to extract the realistic $N N$ potentials. This will be indeed started soon as a first priority simulation at 10 PFlops national " $\mathrm{K}$ " supercomputer which will have full operation in 2012 at Advanced Institute for Computational Science (AICS) in Kobe, Japan [32]. Simulations of the three or more nucleons on the lattice are also a challenging problem to be studied in relation to the attractive binding of finite nuclei and to the repulsive effect in high density matter relevant to neutron stars. Study along this line has been recently started [33, 34].

If it turns out that the program described in this article indeed works in lattice QCD with the physical quark mass, it would be a major step toward the understanding of atomic nuclei and 
neutron stars from the fundamental law of the strong interaction, the quantum chromodynamics.

Note added: Recently, full QCD simulations of the hyperon potentials in the flavor SU(3) limit reported in [25] and discussed in Sec. 4 were extended to the lattice sizes $L \simeq 3$ and $4 \mathrm{fm}$ for the pseudo-scalar meson mass of 673-1015 MeV. By solving the Schrödinger eqaution with the flavor-singlet potential, a bound $H$-dibaryon with the binding energy of $30-40 \mathrm{MeV}$ was found [35] (see also [36]). Since the binding energy turns out to be insensitive to the quark masses, there may be a possibility of weakly bound or resonant $H$-dibaryon even in the real world with lighter quark masses and with the flavor $\mathrm{SU}(3)$ breaking. To make a definite conclusion, however, the (2+1)-flavor lattice QCD simulations for $H$-dibaryon with $\Lambda \Lambda-N \Xi-\Sigma \Sigma$ coupled channel analysis is necessary. Such a direction is currently in progress [37].

\section{Acknowledgements}

This work was supported by Grant-in-Aid for Scientific Research on Innovative Areas (No. 2004: 20105001,20105003). I thank the members of HAL QCD collaboration (S. Aoki, T. Doi, Y. Ikeda, T. Inoue, N. Ishii, K. Murano, H. Nemura, K. Sasaki) for fruitful discussions. Also, I thank Y. Fujiwara, C. Nakamoto, M. Oka, T. Takatsuka, R. Tamagaki and H. Toki for stimulating discussions and encouragements.

\section{References}

[1] http://nn-online.org/

[2] G. E. Brown, T. T. S. Kuo, J. W. Holt and S. Lee, The Nucleon-Nucleon Interaction and the Nuclear Many-Body Problem (World Scientific, Singapore, 2010).

[3] G. E. Brown and A. D. Jackson, Nucleon-nucleon Interaction (North-Holland, Amsterdam, 1976). R. Machleidt and I. Slaus, J. of Phys. G 27, R69 (2001).

[4] H. Yukawa, Proc. Math.-Phys. Soc. Jpn. 17, 48 (1935).

[5] R. Jastrow, Phys. Rev. 81, 165 (1951).

[6] Y. Nambu, Phys. Rev. 106, 1366 (1957).

[7] R. Tamagaki, Soryushiron Kenkyu (Kyoto) 40, D26 (1969); Prog. Theor. Phys. 44, 905 (1970). M. Hoffberg et al., Phys. Rev. Lett. 24, 775 (1970).

[8] R. Machleidt, arXiv:0704.0807 [nucl-th].

[9] G. Colangelo et al., arXiv:1011.4408 [hep-lat].

[10] M. Lüscher, Nucl. Phys. B 354, 531 (1991).

[11] M. Fukugita et al., Phys. Rev. D 52, 3003 (1995) [arXiv:hep-lat/9501024]. S. R. Beane et al., Phys. Rev. Lett. 97, 012001 (2006) [arXiv:hep-lat/0602010].

[12] N. Ishii, S. Aoki and T. Hatsuda, Phys. Rev. Lett. 99, 022001 (2007) [nucl-th/0611096].

[13] S. Aoki, T. Hatsuda and N. Ishii, Comput. Sci. Dis. 1, 015009 (2008) [arXiv:0805.2462 [hep-ph]].

[14] S. Aoki, T. Hatsuda and N. Ishii, Prog. Theor. Phys. 123, 89 (2010) [arXiv:0909.5585 [hep-lat]]. 
[15] T. Koehler, K. Goral and P. S. Julienne, Rev. Mod. Phys. 78 1311, (2006) [cond-mat/0601420].

[16] K. Nishijima, Phys. Rev. 111, 995 (1958).

W. Zimmermann, Nuovo Cim. 10, 597 (1958).

R. Haag, Phys. Rev. 112, 669 (1958).

See also, W. Zimmermann, MPI-PAE/PTh-61/87 (1987), unpublished.

[17] W. Królikowski and J. Rzewuski, Nuovo Cimento, 4, 1212 (1956).

[18] R. Tamagaki and W. Watari, Prog. Theor. Phys. Suppl. No. 39, 23 (1967).

[19] S. Okubo and R. E. Marshak, Ann. of Phys. 4, 166 (1958).

[20] S. Aoki et al. (CP-PACS Collaboration), Phys. Rev. D 71, 094504 (2005).

[21] S. Aoki et al. (PACS-CS Collaboration), Phys. Rev. D 79, 034503 (2009) [arXiv:0807.1661 [hep-lat]].

[22] N. Ishii (for PACS-CS Collaboration and HAL-QCD Collaboration), PoS LAT2009, 019 (2009) [arXiv:1004.0405 [hep-lat]].

[23] K. Murano, N. Ishii, S. Aoki and T. Hatsuda, arXiv:1012.3814 [hep-lat]; arXiv:1103.0619 [hep-lat].

[24] CP-PACS and JLQCD Collaborations, http://www.jldg.org/ildg-data/CPPACS+JLQCDconfig.html

[25] T. Inoue et al. (HAL QCD collaboration), Prog. Theor. Phys. 124, 591 (2010) [arXiv:1007.3559 [hep-lat]].

[26] R. L. Jaffe, Phys. Rev. Lett. 38, 195 (1977) [Erratum-ibid. 38, 617 (1977)].

[27] M. Oka, K. Shimizu and K. Yazaki, Nucl. Phys. A 464, 700 (1987); Prog. Theor. Phys. Suppl. 137, 1 (2000).

Y. Fujiwara, Y. Suzuki and C. Nakamoto, Prog. Part. Nucl. Phys. 58, 439 (2007)

[arXiv:nucl-th/0607013]; Y. Fujiwara and Y. Suzuki, Nucl. Phys. News 18N2, 17 (2008).

[28] S. Otsuki, M. Yasuno and R. Tamagaki, Prog. Theor. Phys. Suppl. Extra Number 1965, 578 (1965).

S. Machida and M. Namiki, Prog. Theor. Phys. 33, 125 (1965).

V. G. Neudachin, Yu. F. Smirnov and R. Tamagaki, Prog. Theor. Phys. 58, 1072 (1977).

[29] T. Kawanai and S. Sasaki, Phys. Rev. D 82, 091501 (2010) [arXiv:1009.3332 [hep-lat]]; arXiv:1011.1322 [hep-lat].

[30] Y. Ikeda (for HAL QCD Collaboration), arXiv:1002.2309 [hep-lat].

[31] H. Nemura, N. Ishii, S. Aoki and T. Hatsuda, Phys. Lett. B 673, 136 (2009) [arXiv:0806.1094 [nucl-th]].

H. Nemura (for HAL QCD and PACS-CS Collaborations), PoS LAT2009, 152 (2009) [arXiv:1005.5352 [hep-lat]].

[32] http://www.aics.riken.jp/index.html

[33] T. Yamazaki, Y. Kuramashi, A. Ukawa, Phys. Rev. D 81, 111504 (2010) [arXiv:0912.1383 [hep-lat]].

[34] T. Doi (for HAL QCD Collaboration), arXiv:1011.0657 [hep-lat].

[35] T. Inoue et al. [HAL QCD Collaboration], Phys. Rev. Lett. bf 106, 162002 (2011) [arXiv:1012.5928 [hep-lat]].

[36] S. R. Beane et al. (NPLQCD Collaboration), Phys. Rev. Lett. bf 106, 162001 (2011) [arXiv:1012.3812 [hep-lat]].

[37] K. Sasaki (for HAL QCD Coll.), PoS LAT 2010, 157 (2010). 